Supporting Information of

\title{
Tunable Terahertz Metamaterials Based on Anapole Excitation with Graphene for Reconfigurable Sensors
}

Xiaoyu Liu ${ }^{\dagger}$, Zizheng Liu ${ }^{\dagger}$, Ming Hua ${ }^{\dagger}$, Luyao Wang ${ }^{\dagger}$, Kaifeng Wang ${ }^{\dagger}$, Weiwei Zhang ${ }^{\dagger}$, Yafei Ning ${ }^{\dagger}$, Yanpeng Shi ${ }^{*}{ }^{\dagger}$, Xiaodong Wang ${ }^{\star}$ and Fuhua Yang

tCenter of Nanoelectronics and School of Microelectronics, Shandong University, Jinan 250100, China

*Engineering Research Center for Semiconductor Integrated Technology, Institute of Semiconductors, Chinese Academy of Sciences, Beijing 100083, China

Corresponding Author: Yanpeng Shi

E-mail: ypshi@sdu.edu.cn 


\section{The electromagnetic parameter of the placed graphene}

Surface conductivity $\sigma$ can be used to characterize for the optical feature of monolayer graphene, which is given by:

$$
\begin{gathered}
\sigma\left(\omega, \Gamma, \mu_{c}, \mathrm{~T}\right)=\sigma_{\text {int } r a}\left(\omega, \Gamma, \mu_{c}, \mathrm{~T}\right)+\sigma_{\text {inter }}\left(\omega, \Gamma, \mu_{c}, \mathrm{~T}\right) \\
\sigma_{\text {int } r a}\left(\omega, \Gamma, \mu_{c}, \mathrm{~T}\right)=\frac{-\mathrm{i} e^{2}}{\pi \mathrm{d}^{2}(\omega+i 2 \Gamma)} \int_{0}^{\infty} \xi\left(\frac{\partial f_{d}(\xi)}{\partial \xi}-\frac{\partial f_{d}(-\xi)}{\partial \xi}\right) d \xi \\
\sigma_{\text {inter }}\left(\omega, \Gamma, \mu_{c}, \mathrm{~T}\right)=\frac{i e^{2}(\omega+i 2 \Gamma)}{\pi \mathrm{d}^{2}} \int_{0}^{\infty} \frac{f_{d}(\xi)}{(\omega+i 2 \Gamma)^{2}}-\frac{f_{d}(-\xi)}{4(\xi / \square)^{2}} d \xi \\
\mathrm{f}_{d}(\xi)=\frac{1}{\exp \left(\left(\xi-\mu_{c}\right) /\left(k_{B} \mathrm{~T}\right)\right)+1}
\end{gathered}
$$

where $\omega$ is the angular frequency, $\Gamma$ is the scattering rate, $\mu_{\mathrm{c}}$ is the chemical potential, $\mathrm{T}$ is the temperature, $\mathrm{e}$ is the electron charge, $\mathrm{h}$ is the reduced Plank constant, $\mathrm{kB}$ is the Boltzmann constant, $\xi$ is the energy, and $f_{d}$ is the Fermi-Dirac distribution. The two conductivity terms in (2) and (3) are referred to as the intraband and interband terms, respectively.
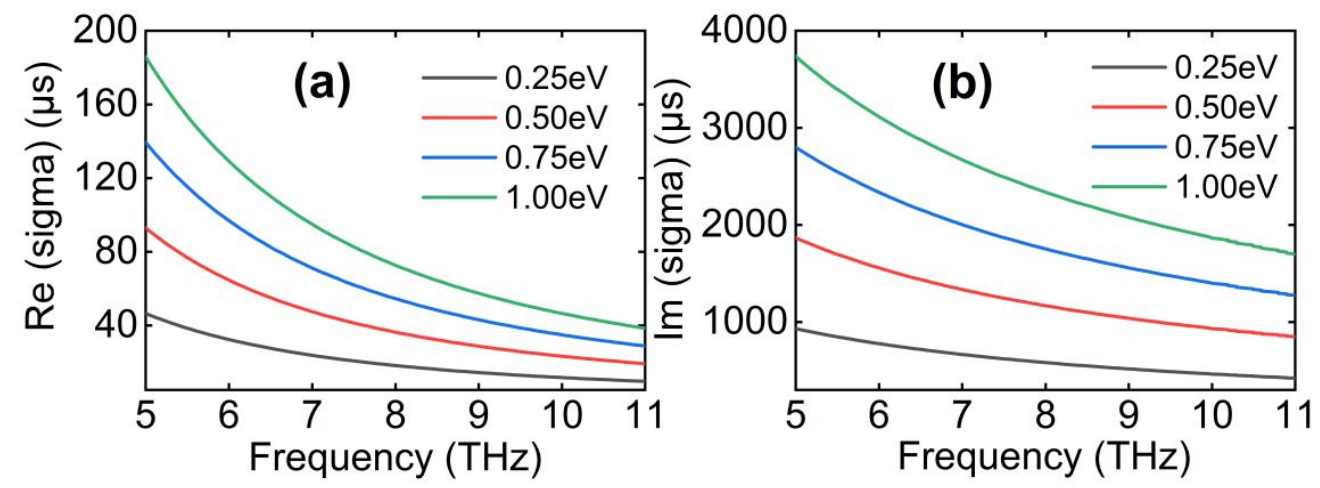

Figure S1. (a) The real part and (b) the imaginary part of the frequency dependent graphene conductivity at the selected values of the Fermi energy. 


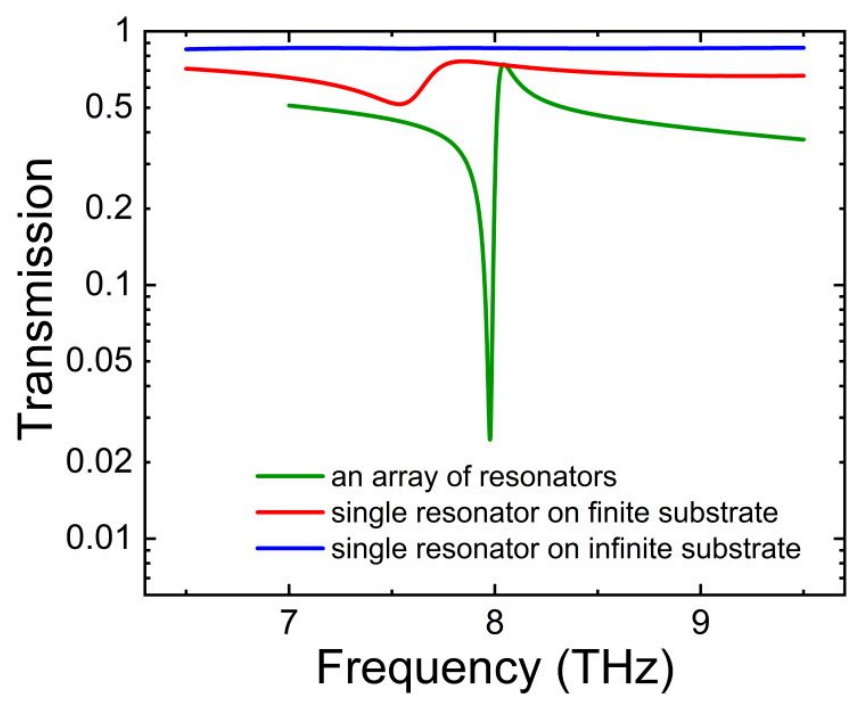

Figure S2. Transmittance spectra of an array of resonators, the single resonator on finite and infinite substrate, respectively. Based on the calculated results, high Q-factor disappeared after the boundary condition was set to unit cell boundary condition. When set as unit cell boundary condition, the single resonator only affects the electromagnetic field around it which results in low Q-factor, the red line shown in Fig. S2. The resonance disappears if the single resonator is displaced on infinite substrate, the blue line shown in Fig. S2. The size of single resonator is smaller than $20 \mu \mathrm{m}$ and the substrate larger than $200 \mu \mathrm{m}$ can be seen as infinite according to the results. It can be seen that high Q-factor will not happen if the resonator is not in an array. ${ }^{1}$ 


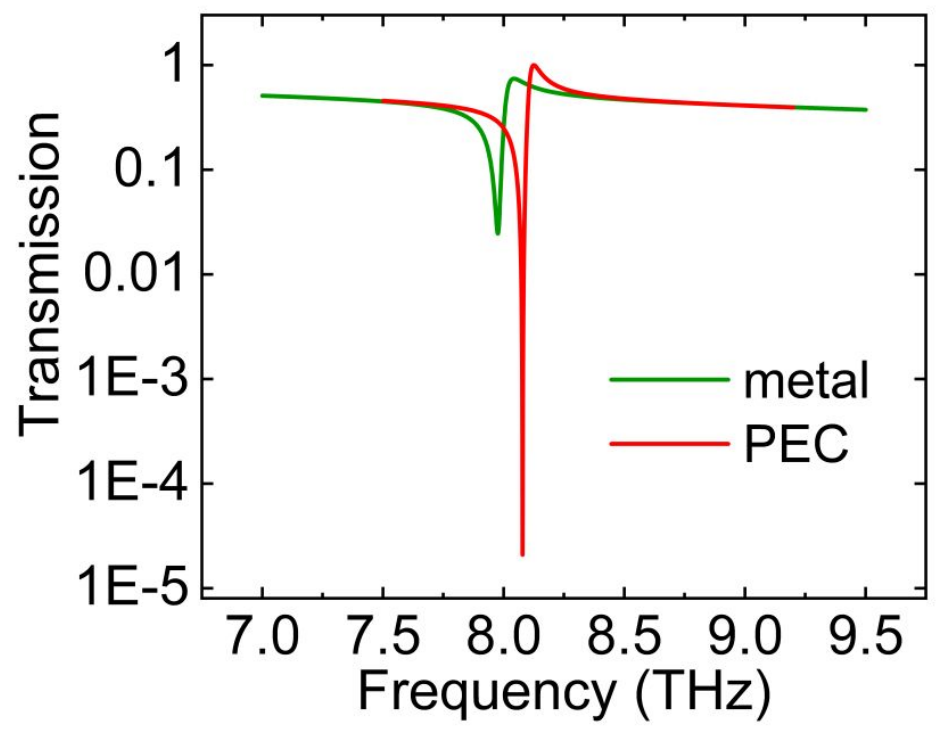

Figure S3. Transmittance spectra of the proposed structure with metal and perfect electric conductor (PEC) resonators, respectively. To studied the effort of non-radiative loss in the terahertz devices, we utilized PEC to replace noble metals in the study, which can largely suppress the non-radiative loss. The resonance frequency has a little blueshift. The resonance dip can exceed 1E-4 without non-radiative loss. And the corresponding Q-factor is up to about $\sim 4.04 \times 10^{4}$. 

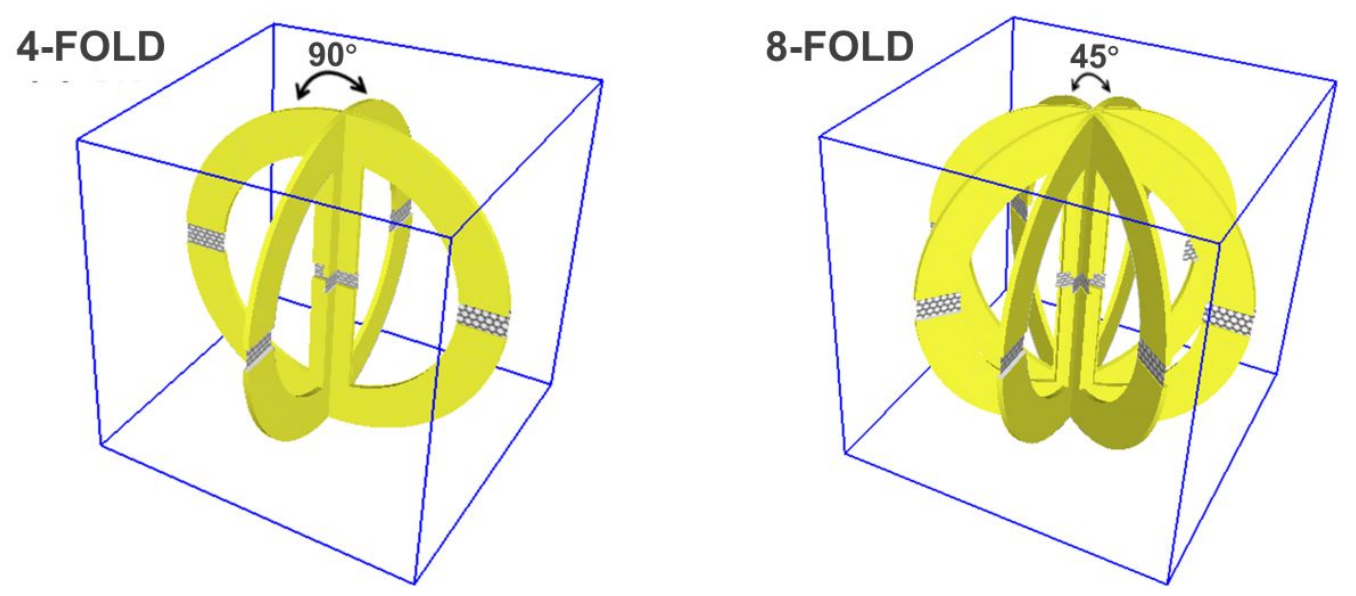

Figure S4. A schematic diagram of 4-fold and 8-fold symmetric meta-molecule. In reality, an electromagnetic wave incident onto a planar metamaterial, would induce higher order multipoles other than the electric and the toroidal dipole. The pair of counter-rotating currents resulted from the proposed structure, that lead to the toroidal dipole, would also lead to the magnetic quadrupole. The scattering contribution from the magnetic quadrupole can be effectively suppressed in the proposed metamaterialbased structure of higher rotational symmetry such as, 4-fold or 8-fold symmetric meta-molecules. $^{2}$ An 8-fold symmetric meta-molecules consists of four meta-molecules with the angle of 45 degree other than 90 degree. 


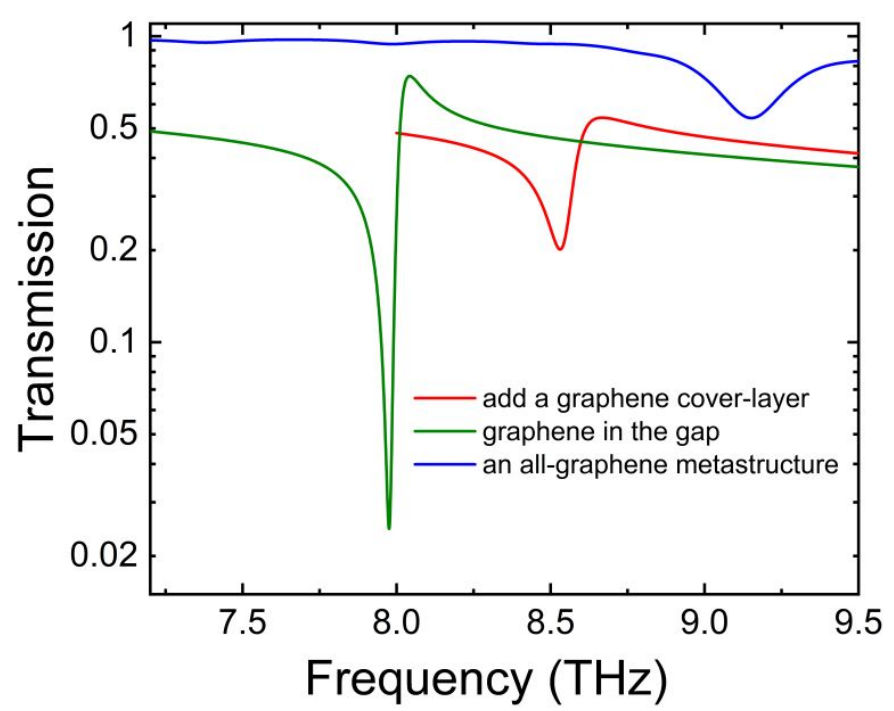

Figure S5. Transmittance spectra of a graphene cover-layer structure, an all-graphene metastructure and our proposed structure, respectively. A graphene cover-layer degrades the tunability performance. For an all-graphene metastructure, graphene results more attractive as a reconfigurable media providing tunability rather than as a reconfigurable plasmonic material by itself at terahertz frequencies. Graphene/metal hybrid structures can offer stronger resonances, larger quality factors, and less sensitivity to the graphene quality than the graphene-only plasmonic structures. ${ }^{3-5}$

\section{REFERENCES}

(1) Li, S. Q.; Zhou, W.; Buchholz, D. B.; Ketterson, J. B.; Ocola, L. E.; Sakoda, K.; Chang, R. P. H. Ultra-sharp plasmonic resonances from monopole optical nanoantenna phased arrays. Applied Physics Letters 2014, 104, 231101.

(2) Savinov, V. Novel Toroidal and Superconducting Metamaterials. University of Southampton, 2014, 79-87.

(3) Zhang, Y.-Q.; Li, J.; Jin, R.-C.; Li, J.-Q.; Wang, J.; Dong, Z.-G. Tunable Terahertz Toroidal Response by Graphene Metamaterials. Mater. Res. Express 2019, 6, 075805. 
(4) Emani, N. K.; Chung, T. F.; Ni, X.; Kildishev, A. V.; Chen, Y. P.; Boltasseva, A. Electrically Tunable Damping of Plasmonic Resonances with Graphene. Nano Lett. 2012, 12, 5202-5206.

(5) Arezoomandan, S.; Quispe, H. O. C.; Ramey, N.; Nieves, C. A.; Sensale-Rodriguez, B. Graphene-based reconfigurable terahertz plasmonics and metamaterials. Carbon 2017, 112, 177-184. 